\title{
A Universal Equation of State for Hydrocarbons
}

\author{
Yosuke KATAOKA $^{\mathrm{a}^{*}}$ and Yuri YAMADA ${ }^{\mathrm{b}, \mathrm{c}}$
}

${ }^{a}$ Department of Chemical Science and Technology, Faculty of Bioscience and Applied Chemistry, Hosei University,

3-7-2 Kajino-cho, Koganei, Tokyo 184-8584, Japan

${ }^{\mathrm{b}}$ Division of Liberal Arts, School of Science and Engineering, Tokyo Denki University,

Hatoyama, Hiki-gun, Saitama 350-0394, Japan

${ }^{\mathrm{c}}$ Research Center for Computing and Multimedia Studies of Hosei University,

3-7-2 Kajino-cho, Koganei, Tokyo 184-8584, Japan

*e-mail: yosuke.kataoka.7t@stu.hosei.ac.jp

(Received: October 31, 2014; Accepted for publication: January 13, 2015; Online publication: February 26, 2015)

A simple, universal equation of state (EOS) for hydrocarbons is proposed. This EOS allows estimation of the effective potential parameters $\varepsilon$ and $\sigma$ in the Lennard-Jones function as analytic functions of $n$, the number of electrons in the hydrocarbon molecule. Using these parameters, the thermodynamic properties of hydrocarbons may be calculated based on the EOS for a perfect liquid, as already proposed in the Lennard-Jones system. The thermodynamic properties estimated in this manner for various hydrocarbons have been found to be reasonably similar to experimentally obtained values.

Keywords: Critical Point, Boiling Temperature, Vaporization Enthalpy, Triple Point, Lennard-Jones System

\section{Introduction}

The critical constants associated with a gas-liquid transition are important as a means of characterizing a fluid system [1]. The experimentally-obtained critical temperatures, $T_{c}$, of a series of hydrocarbons $[2,3]$ are plotted as a function of the number of electrons, $n$, in the molecules in Figure 1. Figures 2 and 3

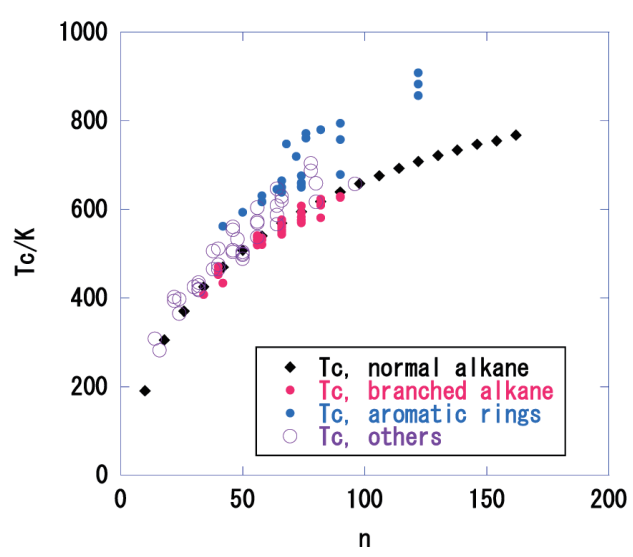

Figure 1. Experimentally obtained critical temperature values vs. the number of electrons in various hydrocarbons $[2,3]$. plot the critical pressure, $p_{\mathrm{c}}$, and the critical volume, $V_{\mathrm{c}}$, in the same manner, respectively. Molecules are classified into natural groups (normal alkane, branched alkane, aromatic rings and others). A list of these is shown in the Appendix. These plots demonstrate that the critical constants of a hydrocarbon depend on the number of electrons in a relatively simple manner, if some deviations from the general trends are neglected.

In this paper, for the sake of simplicity, the Lennard-Jones (LJ) function [1] will be applied to the analysis of these critical constants. The following equation of state (EOS) for a perfect liquid [4] will be used in the analysis:

$$
\begin{aligned}
U(V, T) & =\frac{3}{2} N k T+U_{\mathrm{e}}(V, 0 \mathrm{~K})+g(v) N k T, \\
p(V, T) & =\frac{N k T}{V}-\frac{d U_{\mathrm{e}}(V, 0 \mathrm{~K})}{d V}+f(v) N k T \\
& +\left(\frac{d g(v)}{d v}\right) N k T \ln (k T), \\
v=\frac{V}{N} &
\end{aligned}
$$

Here, $U, V, T$ and $p$ are the internal energy, volume, tem- 


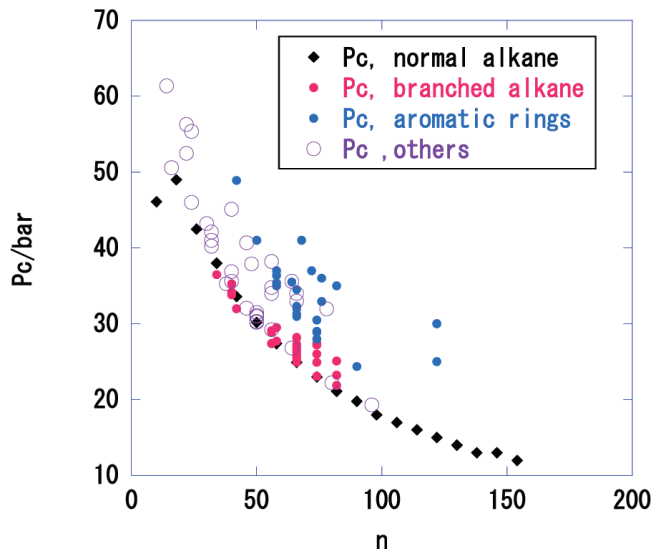

Figure 2. Experimentally obtained critical pressure values vs. the number of electrons in various hydrocarbons [2,3].

perature and pressure of the system, while $k$ is the Boltzmann constant and $N$ is the number of molecules in the system, while the average potential energy is denoted as $U_{\mathrm{e}}$. The functions $f(v)$ and $g(v)$ are polynomials of the volume per particle, $v$, while the final term in the equation $p$ serves as a correction to satisfy the thermodynamic equation of state (EOS) [1] reproducing the three-phase diagram of argon [4]. Although most hydrocarbon molecules are not spherical, the LJ parameters have commonly been applied to the estimation of many such molecules [5].

In the following sections, the $\mathrm{LJ}$ parameters will be estimated as functions of $n$, following which the calculated critical constants will be compared with experimentally observed results. The boiling, melting and triple point temperatures will all be calculated to compare with actual values, and the estimated enthalpies of vaporization will also be compared with macroscopic data. These comparisons will demonstrate that the EOS for a perfect liquid combined with the $n$-dependent LJ parameters can predict various thermodynamic quantities, at least on a semiquantitative basis.

\section{LJ parameters and critical constants}

In this work, molecular interactions between molecules modeled as spheres were determined based on the LJ potential [1], which is a function of the interatomic distance, $r$, and is given by the following equation:

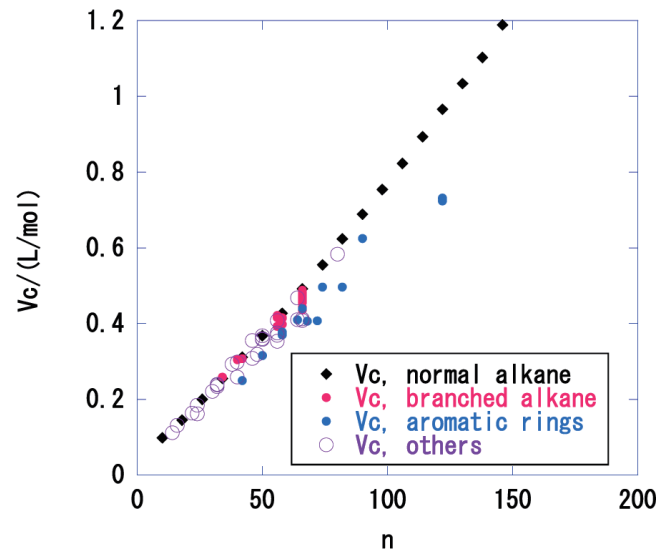

Figure 3. Experimentally obtained critical volumes vs. the number of electrons in various hydrocarbons $[2,3]$.

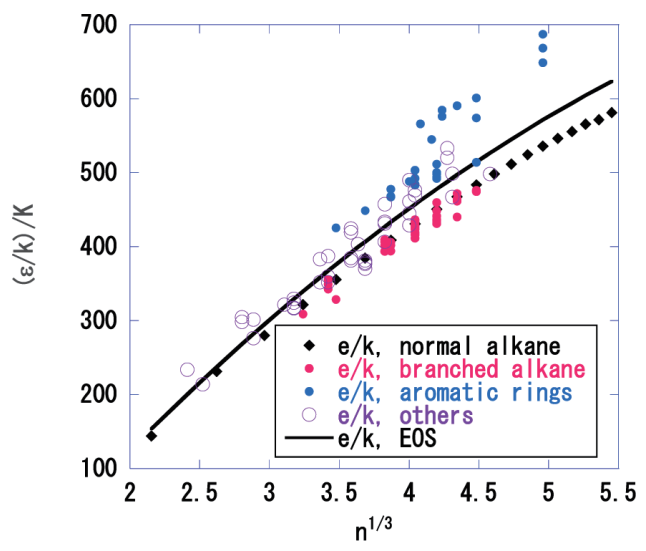

Figure 4. The potential parameter $\varepsilon$ vs. $n^{1 / 3}$ for various hydrocarbons.

$$
u(r)=4 \varepsilon\left[\left(\frac{\sigma}{r}\right)^{12}-\left(\frac{\sigma}{r}\right)^{6}\right]
$$

where $\varepsilon$ is the depth of the potential well and $\sigma$ is the separation at which $u(\sigma)=0$.

The critical temperature and the pressure may then be written in terms of LJ parameters [4] as:

$$
\begin{aligned}
& T_{c}=1.321 \frac{\varepsilon}{k}, \\
& p_{c}=0.219 \frac{\varepsilon}{\sigma^{3}} .
\end{aligned}
$$

Assuming these relationships, the LJ parameters may be obtained from the experimental data for various hydrocarbons [2,3] as shown in Figures 4 and 5, fitted as functions of $n^{1 / 3}$. 


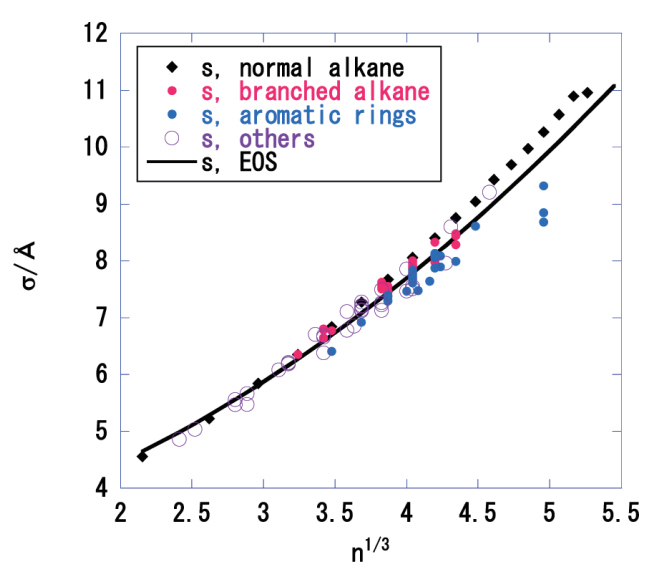

Figure 5. The potential parameter $\sigma$ vs. $n^{1 / 3}$ for various hydrocarbons.

In this least-squares fitting process, second-order polynomials are assumed and, generally speaking, these functions are almost linear with respect to $n^{1 / 3}$. The resulting functions (Equations (4)) are combined with the EOS for a perfect liquid [4] to generate a universal EOS for the hydrocarbons.

$$
\begin{aligned}
& (\varepsilon / k) / \mathrm{K}=-12.865 n^{2 / 3}+240.34 n^{1 / 3}-304.1 ; R^{2}=0.8018 \\
& \sigma / \AA=0.2049 n^{2 / 3}+0.3919 n^{1 / 3}+2.8519 \\
& R^{2}=0.9427
\end{aligned}
$$

Here, $R^{2}$ is the coefficient of determination.

The critical volume may then be written in terms of the above potential parameter as in the following relationship [4].

$$
V_{c}=2.57 \sigma^{3}
$$

The estimated critical constants obtained from the present EOS are compared with the macroscopic values in Figures 6, 7 and 8 , from which it is evident that the critical temperature and pressure are reproduced reasonably well. The calculated critical volume, however, deviates systematically from the experimental values with a relative error of approximately $40 \%$, representing a weakness in the present EOS, since only the number of electrons in the molecule serves as input data for the equation. However, the general increasing trend of the critical volume as a function of $n$ is reproduced and so, in this sense at least, the present EOS still represents a powerful tool for estimating the critical constants.

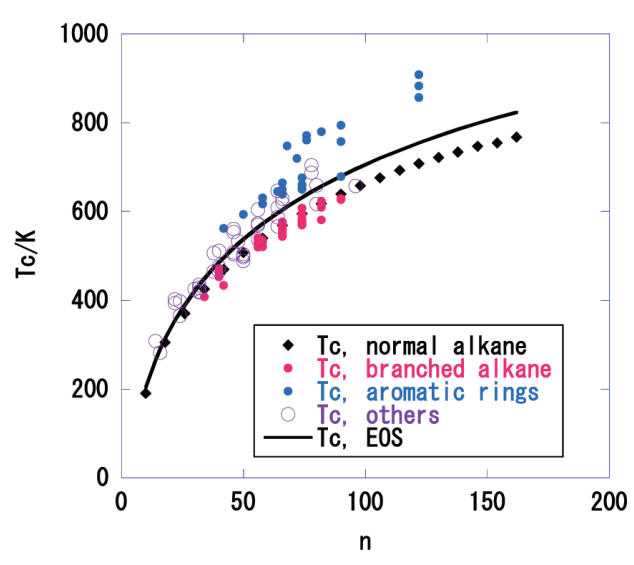

Figure 6. Calculated critical temperature values vs. the number of electrons in various hydrocarbons, including a comparison with experimental data [2,3].

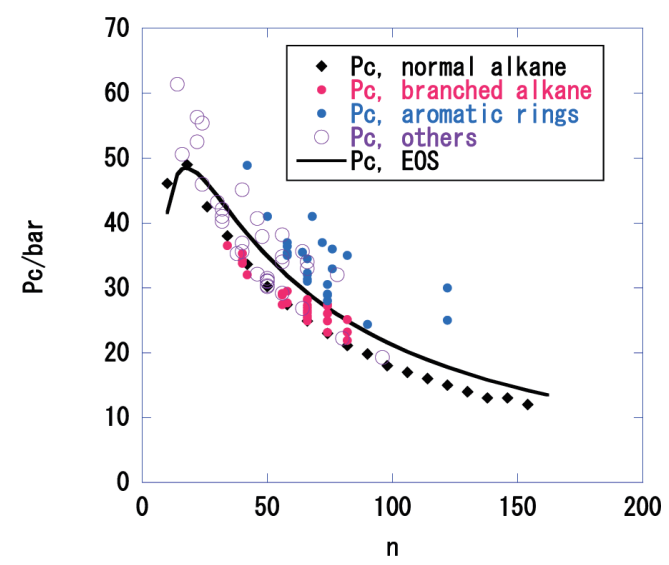

Figure 7. Calculated critical pressure values vs. the number of electrons in various hydrocarbons, including a comparison with experimental data $[2,3]$.

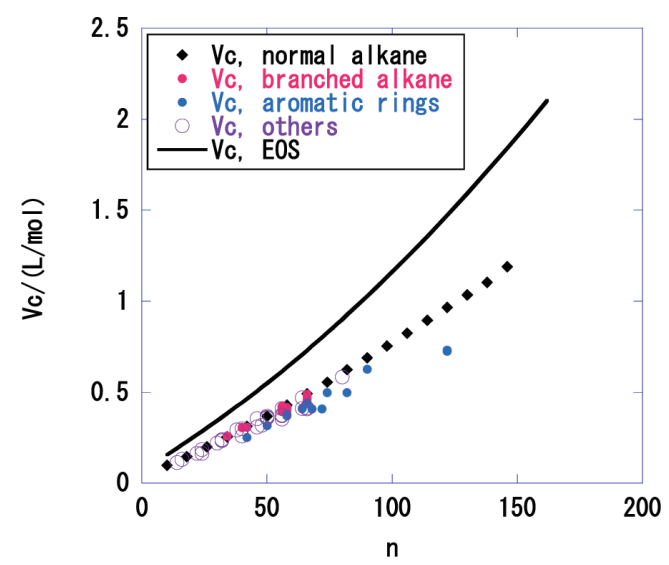

Figure 8. Calculated critical volumes vs. the number of electrons in various hydrocarbons, including a comparison with experimental data [2,3]. 


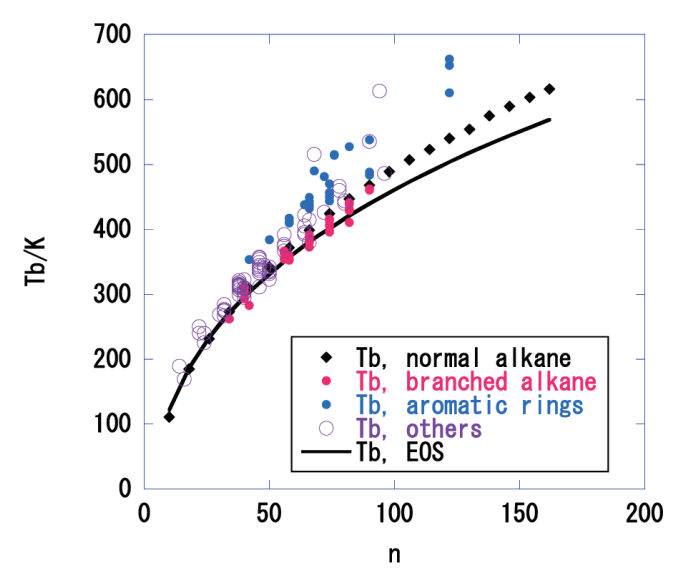

Figure 9. Calculated boiling temperature values vs. the number of electrons in various hydrocarbons, including a comparison with experimental data $[2,3]$.

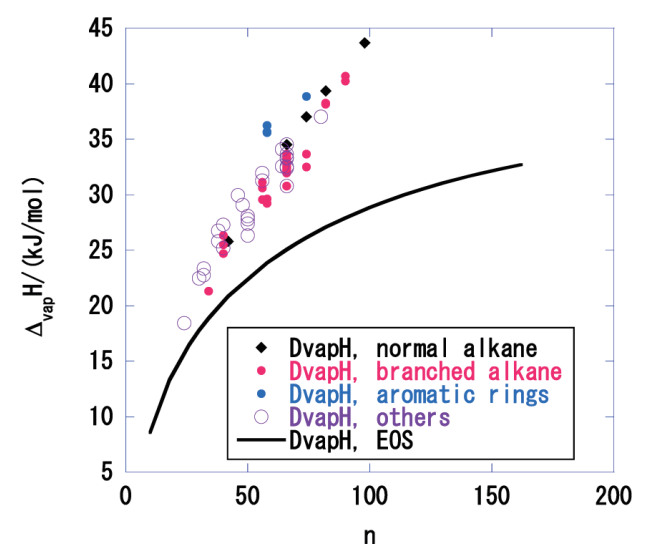

Figure 10. Calculated enthalpy of vaporization values vs. the number of electrons in various hydrocarbons, including a comparison with experimental data $[2,3]$.

\section{Boiling point}

In this section, the boiling point under a pressure, $p$, of $1 \mathrm{~atm}$ is examined. In order to fix the boiling temperature, the following equation was solved numerically for each given value of $n$ :

$$
\begin{aligned}
& p_{g}\left(V_{g}, T\right)=p_{l}\left(V_{l}, T\right)=1 \mathrm{~atm}, \\
& \frac{G_{g}\left(V_{g}, T\right)}{N_{g}}=\frac{G_{l}\left(V_{l}, T\right)}{N_{l}} .
\end{aligned}
$$

An example of the EXCEL worksheet applied to these calculations is provided in the appendix to this text. The resulting boiling temperatures are plotted in Figure 9, and it is evident that the match between these values and the experimental data

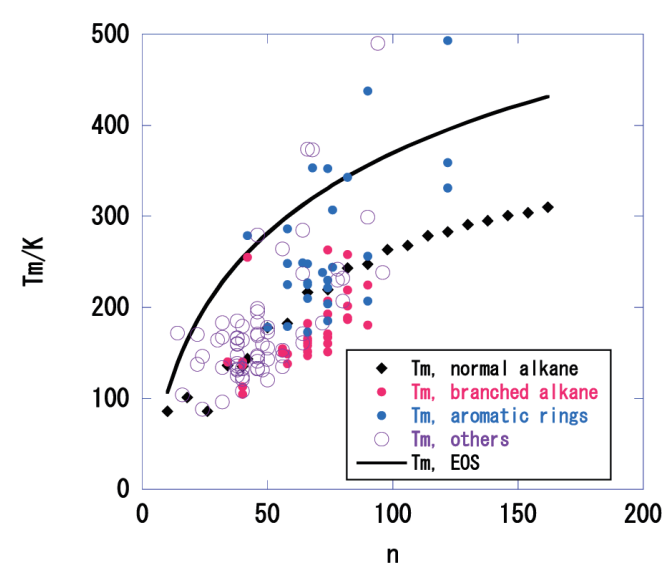

Figure 11. Calculated melting temperature values vs. the number of electrons in various hydrocarbons, including a comparison with experimental data $[2,3]$.

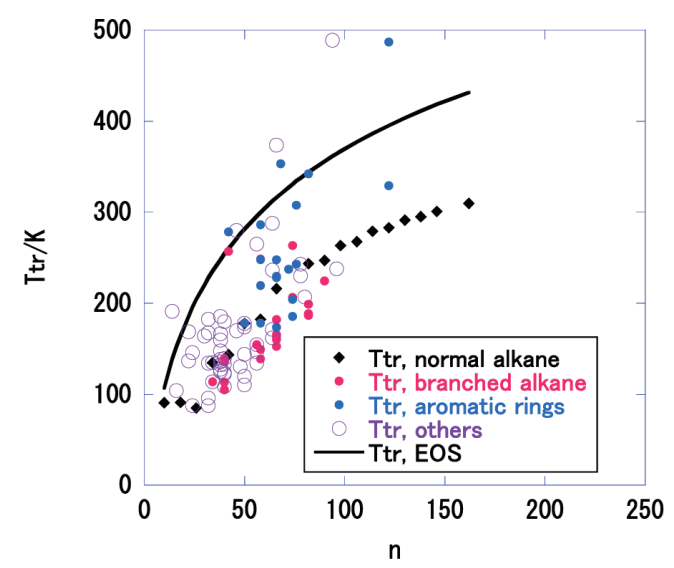

Figure 12. Calculated triple point temperature values vs. the number of electrons in various hydrocarbons, including a comparison with experimental data [2,3].

is satisfactory. The enthalpy of vaporization values are presented in Figure 10. These calculated values are approximately $30 \%$ less than the macroscopic data, although the calculated enthalpies of vaporization do increase as $n$ increases in accordance with the general trend of the experimental results.

\section{$4 \quad$ Melting and triple points}

The EOS for a perfect solid [4] was assumed when estimating the melting and triple point temperatures. Experimental observations indicate that the melting point under a pressure, $p$, of 1 atm is close to the triple point and, for this reason, the estimated melting points were obtained using the triple point of the $\mathrm{LJ}$ system [4]. The experimental melting points are compared with 


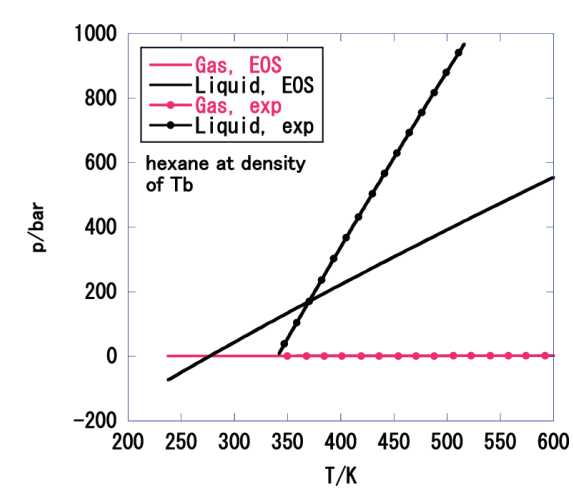

Figure 13. Calculated pressures vs. temperatures for hexane at the density of the boiling point, including a comparison with published data [6].

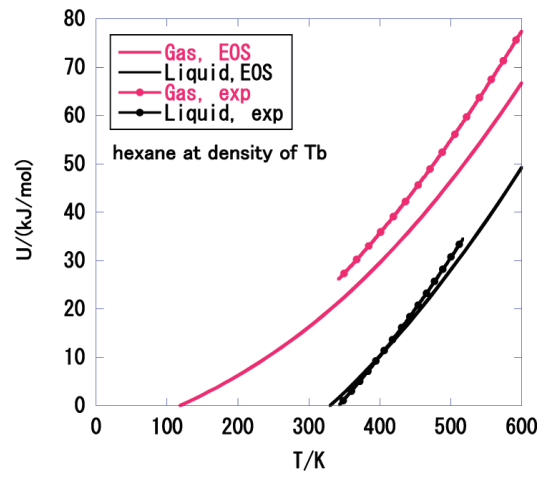

Figure 14. Calculated internal energy values vs. temperature for hexane at the density of the boiling point, including a comparison with published data [6].

Table 1. Density values for hexane at the boiling point (mol/L).

\begin{tabular}{cccc}
\hline $\begin{array}{c}\text { Density(gas), } \\
\exp \end{array}$ & $\begin{array}{c}\text { Density (gas), } \\
\text { EOS }\end{array}$ & $\begin{array}{c}\text { Density (liquid), Density (liquid), } \\
\exp \end{array}$ & \begin{tabular}{c} 
EOS \\
\hline 0.038
\end{tabular} \\
0.043 & 7.113 & 3.770 \\
\hline
\end{tabular}

the calculated values in Figure 11, while a comparison of the triple point data is shown in Figure 12. The EOS values are evidently larger than the experimental values in most cases, possibly because an FCC lattice was assumed for the solid structures in the present EOS and this may be not the case for the majority of solid hydrocarbons. Nevertheless, the present EOS approach is able to provide rough approximations based solely on the number of electrons in the molecule.

\section{$5 p V T$ and $U V T$ relationships at con- stant volume}

In this section, the $p V T$ and $U V T$ relationships are discussed. The $p$ vs. $T$ plot for hexane is shown as a function of temperature at the density of the boiling point in Figure 13, and the pressure is seen to be a linear function of temperature [4]. The slope of the liquid phase pressure line obtained from the EOS results is different from that for the experimental data because the density is different in both cases, as can be seen in Table 1 .

The $U$ vs. $T$ plot is compared in Figure 14. In this Figure the rotational and vibrational contributions are incorporated since the EOS for the perfect liquid includes only translational motion [4]. The vibrational frequencies were calculated using the Gaussian 09 program at the HF/631G (d) level [7]. The vibra- tional contribution $\left\langle E_{\mathrm{v}}>\right.$ is calculated as follows [1]:

$$
\begin{aligned}
\left\langle E_{v}\right\rangle & =N \sum_{i=1}^{N_{v}}\left\langle E_{i}\right\rangle, \\
\left\langle E_{i}\right\rangle & =\frac{\sum_{v=0}^{\infty} E_{i}(v) \exp \left(-E_{i}(v) / k T\right)}{\sum_{v=0}^{\infty} \exp \left(-E_{i}(v) / k T\right)}
\end{aligned}
$$

Here, $N_{v}$ is the number of vibrational modes in the molecule, while $v$ is the quantum number of the vibration. Since the vibrational energy levels are discrete, the internal energy exhibits curvature when plotted as a function of temperature. The comparison of the derived and experimental UVT relationships in Figure 14 shows that the agreement between the two is satisfactory.

\section{$6 \quad p V T$ and $U V T$ relationships at con- stant temperature}

The $p v T$ and the $U V T$ relationships for methane were studied at the following temperatures:

$$
T / T_{c}=1,0.8 \text { and } 0.6
$$

The $p V T$ and $U V T$ results are shown in Figures 15 and 16 . At $T / T_{c}=0.8$ and 0.6 , the pressures in the metastable and the 


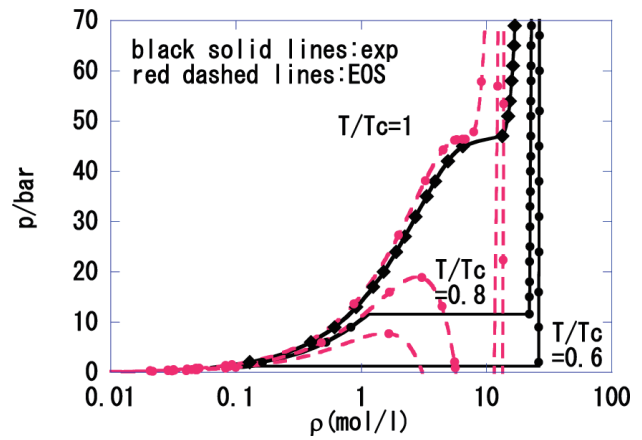

Figure 15. Calculated pressures vs. density for hexane at several temperatures, including a comparison with published data [8].

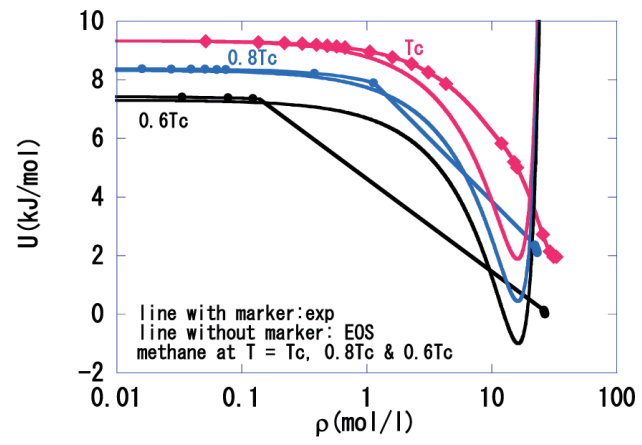

Figure 16. Calculated internal energy values vs. density for hexane at several temperatures, including a comparison with published data [8].

unstable state were also plotted in the region $P>0$ for the EOS. In the gas-phase, the comparison shows adequate agreement, although the EOS pressure increases at relatively low density compared to the experimental data as the density increases. The density of liquid is compared in Table 2.

\section{Discussion}

The present LJ parameters are compared with published values

[5] in Figures 17 and 18.

It is evident that our $\varepsilon$ values are slightly less than those obtained by Cuadros et al. [5], while our values for the size pa-

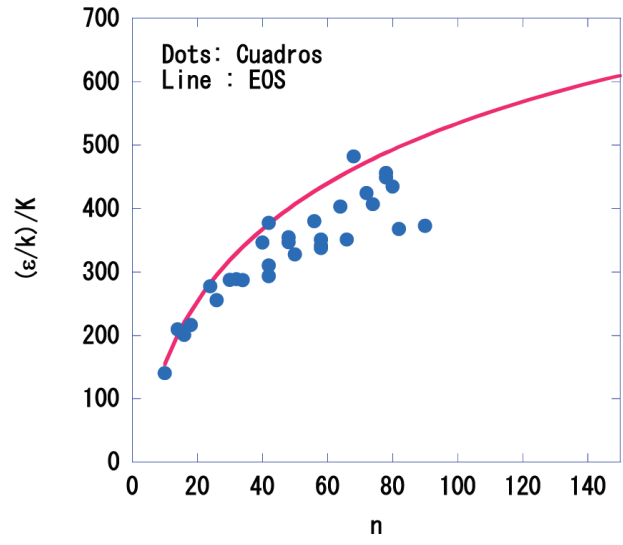

Figure 17. Calculated potential parameter $(\varepsilon)$ values vs. the number of electrons in various hydrocarbons, including a comparison with published data [5].

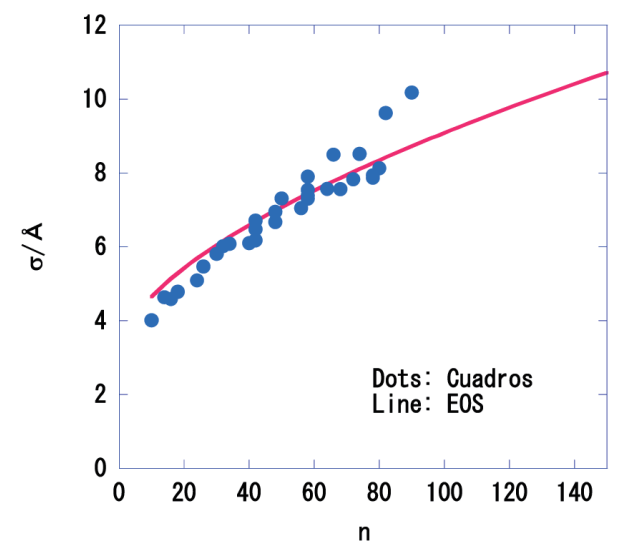

Figure 18. Calculated potential parameter $(\sigma)$ values vs. the number of electrons in hydrocarbons, including a comparison with published data [5].

rameter, $\sigma$, compare satisfactorily, with the exception of those molecules for which $n$ is around 100. This may have occurred because we assumed a pure LJ system, whereas Cuadros et al. [5] applied a different EOS containing an acentric factor [9].

The anisotropic interactions between the hydrocarbon molecules are expected to be averaged by the thermal motion in the gas and liquid phases. As a result, the molecular system may

Table 2. Comparison of density values for methane at liquid-vapor equilibrium (mol/L).

\begin{tabular}{ccccc}
\hline \multirow{2}{*}{$\boldsymbol{T} / \boldsymbol{T} \boldsymbol{c}$} & $\begin{array}{c}\text { Density (gas), } \\
\text { exp }\end{array}$ & $\begin{array}{c}\text { Density (gas), } \\
\text { EOS }\end{array}$ & $\begin{array}{c}\text { Density (liquid), Density (liquid), } \\
\text { exp }\end{array}$ & EOS \\
\hline 0.8 & 1.14 & 1.00 & 21.99 & 11.67 \\
0.6 & 0.14 & 0.12 & 26.08 & 13.44 \\
\hline
\end{tabular}




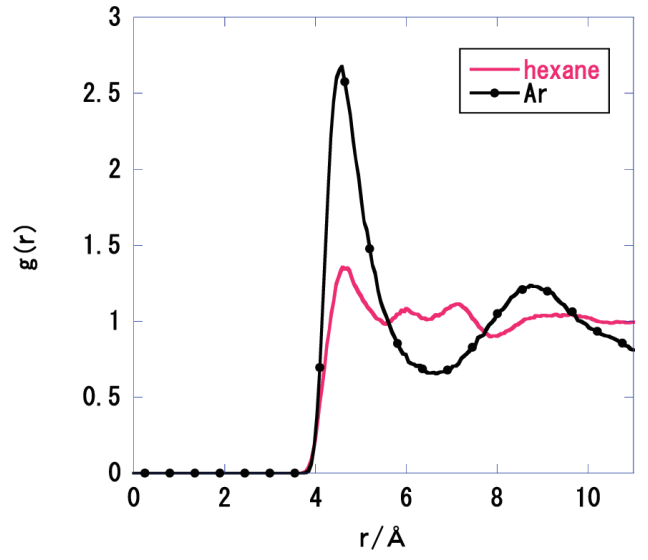

Figure 19. The pair correlation functions of liquid hexane and liquid argon as calculated by molecular dynamics simulations. The correlation between the center of mass is shown.

be considered to be similar to a LJ system, and this possibility was examined by molecular dynamics simulations of hexane. In these computations, a basic cubic cell was employed, containing 500 hexane molecules, and rigid bodies were assumed when solving the equation of motion. The time increment was 1 fs and 100,000 steps were solved using an NTP ensemble, in which $T$ was $300 \mathrm{~K}$ and $p$ was $1 \mathrm{~atm}$, employing the Fujitsu SCIGRESS-ME software package [10]. The OPLS molecular interaction function was assumed [11-14]. The molecular pair correlation function, $g(r)$, in the liquid phase is shown as a function of molecular distance, $r$, in Figure 19, which demonstrates the correlation between the centers of mass. The first peak is found at $r=6 \AA$, and has a width of $2 \AA$ and three sub-peaks. The coordination number of this peak is approximately 10 , while, in the case of liquid argon, this number is about 12 . The second peak is found at approximately $r=10 \AA$, a distance that is close to twice the location of the first peak. This relationship between peaks is frequently seen in simple liquids. The pair correlation function in liquid $\arg o n$ at $\mathrm{T}=100 \mathrm{~K}$ and $P=1 \mathrm{~atm}$ is also shown in Figure 19, although in the case of these data, the $r$ axis for the argon data has been modified such that $r^{\prime}=$ $(11 / 9) r$.

The variation of $\sigma$ with $n$ may be readily explained; the molecular volume of a hydrocarbon is determined almost exclusively by the volume occupied by its carbon atoms. For this reason, the molecular volume as calculated by the Gaussian algorithm at the HF/6-31G (d) level [7] is almost entirely pro-

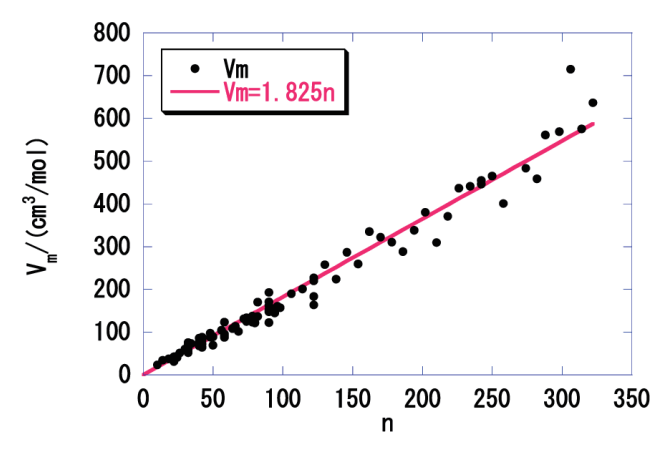

Figure 20. The molecular volume of various hydrocarbons as calculated using molecular orbital theory [7].

portional to $n$, as shown in Figure 20. However, the variation of $\varepsilon$ with $n$ is not as easy to explain. Because the variation of $\varepsilon$ with $n$ is already obtained by the analysis of the experimental data with EOS, we can propose a possible cause. The molecular interaction energy is the sum of repulsive and attractive forces, and these two are balanced near the molecular distance equal to the molecular diameter, as in the case of the LJ model. The repulsive part may be understood as resulting from the Coulomb interaction [1] between the two charges, $q_{1}$ and $q_{2}$, of the two molecules:

$$
u(r)=\frac{q_{1} q_{2}}{4 \pi \varepsilon_{0} r}
$$

The charges $q_{1}$ and $q_{2}$ are assumed to be functions of the total number of electrons in the hydrocarbon molecule, $n$, and the screening factor, $x$, as follows:

$$
q_{1}=q_{2} \propto x n e, x=n^{-2 / 3}
$$

Here $e$ is the unit charge. By this assumption, $q_{1}$ and $q_{2}$ are proportional to $n^{1 / 3}$. In contrast, the molecular volume of a hydrocarbon is almost proportional to $n$, as shown in Figure 20 and so the molecular distance $r$ in equation. (9) is also proportional to $n^{1 / 3}$. Thus the relationship between the energy parameter $\varepsilon$ and $n$ may be written as follows:

$$
\varepsilon \propto \frac{q_{1} q_{2}}{r} \propto \frac{n^{1 / 3} n^{1 / 3}}{n^{1 / 3}} \approx n^{1 / 3} .
$$




\section{Conclusions}

A universal EOS for hydrocarbons has been proposed. Using the number of electrons in a hydrocarbon molecule, the potential parameters $\varepsilon$ and $\sigma$ may be estimated from equation. (4). With these parameters, the thermodynamic properties are calculated using the EOS for a perfect liquid (Eq. (1)) [4]. The estimated properties thus obtained are evidently reasonable approximations of experimentally-obtained values.

The authors would like to thank the Research Center for Computing and Multimedia Studies of Hosei University for the use of computer resources.

\section{References}

[1] P. W. Atkins, Physical Chemistry, Oxford Univ. Press, Oxford (1998).

[2] CRC Handbook of Chemistry and Physics, Ed. D. R. Lide, CRC Press, Boca Raton (1995).

[3] NIST Chemistry WebBook, http://webbook.nist.gov/ chemistry/ (accessed 2014).

[4] Y. Kataoka, Y. Yamada, J. Comput. Chem. Jpn., 13, 130 (2014). [CrossRef]

[5] F. Cuadros, I. Cachadiña, W. Ahamuda, Mol. Eng., 6, 319 (1996). [CrossRef]

[6] R. Span, "Multiparameter Equations of State - An Accurate Source of Thermodynamic Property Data", Springer, Berlin (2000) 367.
[7] M. J. Frisch, G. W. Trucks, H. B. Schlegel, G. E. Scuseria, M. A. Robb, J. R. Cheeseman, G. Scalmani, V. Barone, B. Mennucci, G. A. Petersson, H. Nakatsuji, M. Caricato, X. Li, H. P. Hratchian, A. F. Izmaylov, J. Bloino, G. Zheng, J. L. Sonnenberg, M. Hada, M. Ehara, K. Toyota, R. Fukuda, J. Hasegawa, M. Ishida, T. Nakajima, Y. Honda, O. Kitao, H. Nakai, T. Vreven, J. A. Montgomery, Jr., J. E. Peralta, F. Ogliaro, M. Bearpark, J. J. Heyd, E. Brothers, K. N. Kudin, V. N. Staroverov, R. Kobayashi, J. Normand, K. Raghavachari, A. Rendell, J. C. Burant, S. S. Iyengar, J. Tomasi, M. Cossi, N. Rega, J. M. Millam, M. Klene, J. E. Knox, J. B. Cross, V. Bakken, C. Adamo, J. Jaramillo, R. Gomperts, R. E. Stratmann, O. Yazyev, A. J. Austin, R. Cammi, C. Pomelli, J. W. Ochterski, R. L. Martin, K. Morokuma, V. G. Zakrzewski, G. A. Voth, P. Salvador, J. J. Dannenberg, S. Dapprich, A. D. Daniels, O. Farkas, J. B. Foresman, J. V. Ortiz, J. Cioslowski, D. J. Fox, Gaussian, Inc., Wallingford CT, 2009.

[8] U. Setzmann, W. Wagner, J. Phys. Chem. Ref. Data, 20, 1061 (1991). [CrossRef]

[9] G. Soave, Chem. Eng. Sci., 27, 1197 (1972). [CrossRef]

[10] http://www.scigress.com/ (accessed 2014).

[11] W. L. Jorgensen, E. R. Laird, T. B. Nguyen, J. TiradoRives, J. Comput. Chem., 14, 206 (1993). [CrossRef]

[12] W. L. Jorgensen, J. M. Briggs, M. L. Contreras, J. Phys. Chem., 94, 1683 (1990). [CrossRef]

[13] J. Pranata, S. G. Wierschke, W. L. Jorgensen, J. Am. Chem. Soc., 113, 2810 (1991). [CrossRef]

[14] W. L. Jorgensen, J. Tirado-Rives, J. Am. Chem. Soc., 110, 1657 (1988). [CrossRef] 